\title{
The Preparation and Experimental Research for Inner Cladding Casting of Al-Al
}

\author{
Zeng Li-kui ${ }^{1}$, Yang Ping ${ }^{1}$, Wang Yajun ${ }^{2}$ \\ ${ }^{1}$ College of Intelligent Manufacturing and Automobile, Chongqing Technology and Business College, Chongqing, China \\ ${ }^{2}$ College of Material Science and Engineering, Chongqing University, Chongqing, China
}

\section{Email address:}

659966203@qq.com (Yang Ping)

\section{To cite this article:}

Zeng Li-kui, Yang Ping, Wang Yajun. The Preparation and Experimental Research for Inner Cladding Casting of Al-Al. Science Discovery. Vol. 5, No. 7, 2017, pp. 515-518. doi: 10.11648/j.sd.20170507.17

Received: November 20, 2017; Accepted: November 23, 2017; Published: December 28, 2017

\begin{abstract}
With the rapid development of science and technology, the unceasing enhancement of component integration degree, and the increasingly demanding of parts using conditions, the part that the traditional metal materials and alloy materials preparation is hard to meet the requirements of the use of some special conditions, so the new composite materials have been paid attention to by the countries all over the world. An casting method for Inner Cladding Casting was introduced. By the use of this method, the Al-Al inner cladding casting which has the transition layer in the middle was prepared, it was indicated that the casting method for inner cladding casting has the feasibility. Through the soaking experiments of three group samples which were Arc Spraying Al layer、 substrate of Al and Inner Cladding Casting of Al-Al, we find out that by 140h soaking experiments for inner cladding casting of Al-Al the porosity change was little and it had shown very good corrosion resistant effect.
\end{abstract}

Keywords: Al-Al, Arc Spraying, Inner Boundary, Cladding

\section{Al-AI内界面包覆型铸件的制备与试验研究}

曾利奎 ${ }^{1}$, 杨平 ${ }^{1}$, 王亚军 ${ }^{\mathbf{2}}$

1智能制造与汽车学院, 重庆工商职业学院, 重庆, 中国

${ }^{2}$ 材料科学与工程学院, 重庆大学, 重庆, 中国

邮箱

659966203@qq.com(杨平)

摘要：随着科学技术的飞速发展、零件集成化程度的不断提高以及零件使用条件的日渐苛刻, 传统金属材料和合金材 料所制备的零件已经很难满足某些特殊工况的使用要求，而新型复合材料的研究已受到世界各国的普遍重视。本文提 出一种用于铸造内界面包覆型铸件的制造工艺, 并由此制备了拥有中间过渡层的Al-Al内界面包覆型铸件, 说明了该铸 造方法的可实施性。经过电弧喷涂 $\mathrm{Al}$ 层、基体 $\mathrm{Al}$ 及 $\mathrm{Al}-\mathrm{Al}$ 内界面包覆型铸件三组试样的 $\mathrm{NaCl}$ 溶液浸泡对比实验, 发现 Al-Al内界面包覆型铸件经过140小时浸泡实验后, 气孔和间隙变化不大, 表现出很好的耐腐蚀效果。

关键词: $\mathrm{Al}-\mathrm{Al}$, 电弧喷涂, 内界面, 包覆 


\section{1. 前言}

在目前进行过的科研实验里，物件的热喷涂技术大多 数是对型材或铸件的外表面进行热喷涂处理以得到目标 性能的外表面涂层, 然而对于型芯、型腔等内界面的热喷 涂处理的技术却鲜有耳闻, 但内界面通常为零件间接合的 地方, 对型材或铸件的综合性能要求较高, 比如传动轴外 径与轴承内径的配合, 就需要具有相互对应特性的接触材 料[1-9]。该实验旨在对型材或型材之间内界面包覆进行研 究, 提出一种经济实用的内界面包覆型铸件的实验方法。 传统喷涂工艺所得到的表面喷涂层间隙比较大, 通常 使用表面封孔处理的方式减少涂层与腐蚀介质之间的接 触面积, 常见的封孔处理工艺包括渗油、涂料封闭、耐高 温封闭及扩散热处理等, 电弧喷涂金属涂层的封闭剂大体 上可以分为不固化型(如油、脂、蜡)、固化型(如环氧类、 乙烯类、聚酷类、厌氧类等双组分固化涂料)及自封闭金 属(如锡、铜、铝)三大类[10-11], 封孔剂对于结构复杂的 零部件封孔效果较差, 且费时费力,不利于大规模生产。另 外, 现有封孔剂大多具有不导电性,因此在一些特殊领域, 这类封孔涂层无法应用。例如电子通讯行业大多要求器件 涂层具有导电、耐腐蚀、抗静电等性能。针对以上问题, 本实验制得的包覆型铸件就迎刃而解, 即使未进行封孔处 理, 也能达到理想效果。

\section{2. 材料制备与方法}

选用纯铝丝作为喷涂材料, 内层金属液为ZL101, 实 验前需对耐火砖制备的型芯进行预热处理以减少喷涂层 的内应力, 预热温度为 $500^{\circ} \mathrm{C}-600^{\circ} \mathrm{C}$, 同时预热模具以降 低内层金属液的凝固速率, 预热温度为 $600{ }^{\circ} \mathrm{C}-700^{\circ} \mathrm{C}$ 。本 实验喷涂时采用的喷涂参数如表 1 所示。用电阻加热炉将 浇注的ZL101进行熔化, 并用覆盖剂进行处理, 当ZL101 温度达到 $750^{\circ} \mathrm{C} \pm 10^{\circ} \mathrm{C}$ 时, 倒出加热炉浇注, 并将带喷涂层 的型芯放入模具中, 最终获得实验所需的 Al-Al内界面包 覆型铸件，其示意图如图1所示。
表1 喷涂工艺参数。

\begin{tabular}{lll}
\hline 影响因素 & 送丝电压 $(\mathbf{V})$ & 喷涂电压 $(\mathbf{V})$ \\
\hline 参数值 & $10-25 \mathrm{~V}$ & $27.9 \mathrm{~V}(29$ 档 $)$ \\
\hline
\end{tabular}

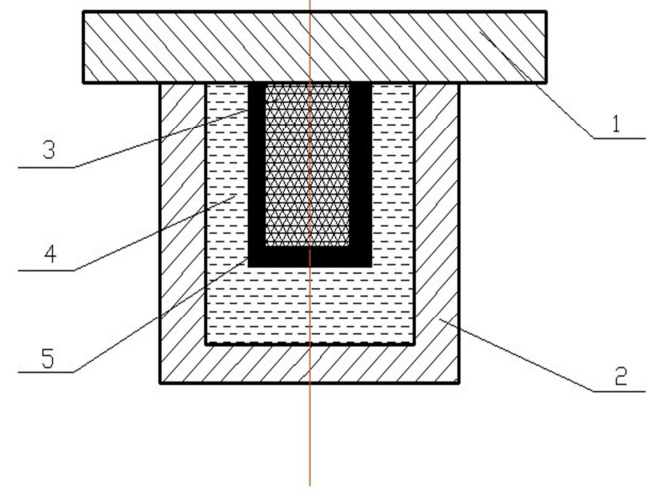

1.型芯支撑板；2.铸型；3.喷涂层；4.金属液；5.型芯

图1 喷涂示意图。

\section{Al-Al内界面包覆型铸件浸泡实验}

对 10-25V的电弧喷涂 $\mathrm{Al}$ 层、表面未经处理的 $\mathrm{Al}$ 基体和 $\mathrm{Al}-\mathrm{Al}$ 包覆型铸件分别进行 $5 \%$ 的 $\mathrm{NaCl}$ 溶液浸泡处理, 并对 三种试样分别编号 $1 、 2 、 3$ 。具体操作为: 将三种材料均 切割成 $20 \mathrm{mmx} 10 \mathrm{mmx} 20 \mathrm{~mm}$ 规格的试样, 将编号为 $1 、 2 、 3$ 的三个试样分别放入具有对应编号为 $1 、 2 、 3$ 的三个烧杯 中, 然后将试样浸没在 $5 \%$ 的 $\mathrm{NaCl}$ 溶液中, 温度保持在 $20^{\circ} \mathrm{C}$ $\pm 5^{\circ} \mathrm{C}$, 随时观察浸泡过程中铸件表面的腐蚀情况, 140小 时后取出试样进行金相分析。实验中, 需控制溶液体积与 试样表面积的比例大于 $20 \mathrm{~mL}: 1 \mathrm{~cm}^{2}$ 以降低腐蚀产物对试 验结果的影响。观察试样随时间变化的情况如表2所示.

表2 浸泡实验。

\begin{tabular}{llll}
\hline 时间 $\mathbf{t} / \mathbf{h}$ & $\mathbf{1 0 - 2 5}$ 涂层 & 表面未处理基体 & Al-Al包覆型铸件 \\
\hline 0 & 无变化 & 无变化 & 无变化 \\
0.5 & 无变化 & 无变化 & 无变化 \\
3 & 无变化 & 无变化 & 无变化 \\
6 & 有气泡出现, 并伴随有啪啪的响声 & 有气泡出现 & 无变化 \\
10 & 气泡增多, 出现少许絮状白色沉淀, 并伴随有啪啪的响声 & 气泡增多, 出现少许絮状白色沉淀 & 无变化 \\
13 & 气泡增多, 出现少许絮状白色沉淀, 响声变小 & 絮状白色沉淀增多 & 无变化 \\
20 & 絮状白色沉淀继续增加, 响声变小 & 絮状白色沉淀继续增多 & 无变化 \\
$20-140$ & 䋈状白色沉淀继续增加, 响声基本消失 & 絮状白色沉淀增加趋势变缓 & 表面变暗 \\
$140-720$ & 试样厚度减小 & 试样厚度减小 & 稍微腐蚀 \\
\hline
\end{tabular}

\section{4. 实验结果与分析}

对浸泡后的三份试样分别进行 400 号砂子粗磨、 1000 号砂子细磨、抛光以及用浓度为 $5 \%$ 的稀硝酸进行腐蚀, 将处理后的试样放在ZEISS Axiovert 200 MAT金相显微镜
下观察，三种试样的金相组织如图2、图3和图4所示。观 察图2可知, 浸泡前的电弧喷涂 $\mathrm{Al}$ 层间隙和气孔较少, 并 散乱分布在试样表面, 而经过 $\mathrm{NaCl}$ 溶液浸泡的试样间隙和 气孔明显增多, 且气孔变大、互相贯通, 大量分布在试样 表面。观察图3和图4可知, 经 $\mathrm{NaCl}$ 溶液处理后, $\mathrm{Al}$ 基体也 呈现出不同程度的腐蚀现象, 而用包覆型铸造方法制得的 
铸件经浸泡处理后截面的间隙和气孔变化很小, 甚至看不 出变化。

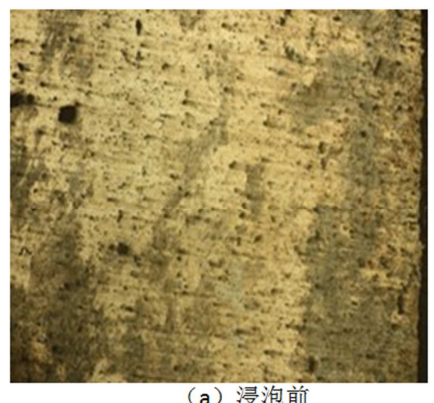

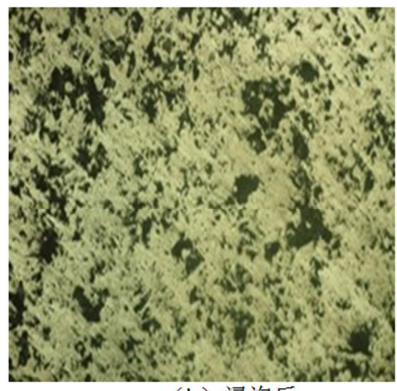

（b）浸泡后
图2 10-25V涂层浸泡实验前后对比图。

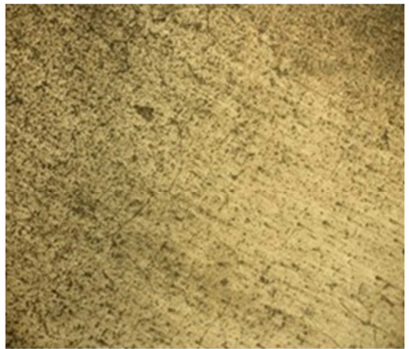

（a）浸泡前

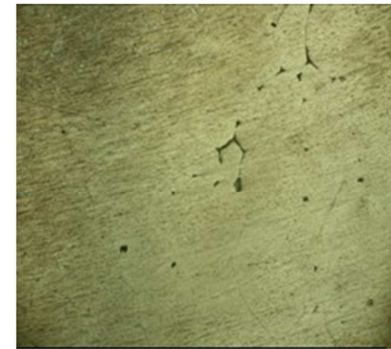

（b）浸泡后
图3 基体浸泡实验前后对比图。

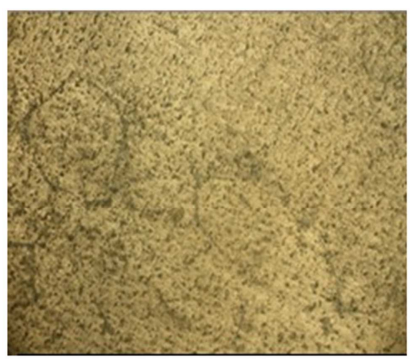

（a）浸泡前

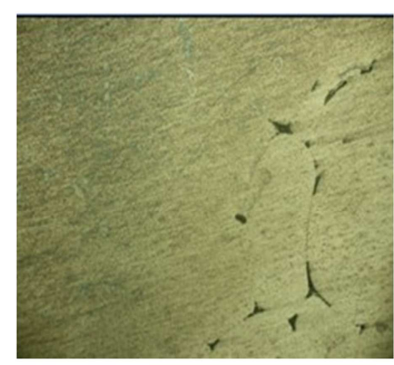

（b）浸泡后
图4 Al-Al包覆铸件浸泡实验前后对比图。

从图2和图3可以看出, 经由 $5 \%$ 的 $\mathrm{NaCl}$ 溶液处理过的 喷涂层和基体, 喷涂外表面均出现腐蚀凹坑, 呈现出显著 的点蚀现象。其原因为: 未经封孔处理的试样表面, 负价 的游离态氯离子能吸附在铝合金氧化膜上, 由于氯离子半 径小且能够透金属氧化膜，从而到达其内部，使氧化膜受 电荷影响, 感应产生出强烈的带电粒子流。产生的带电粒 子使氧化膜在一定程度上维持高电流密度, 并使阳离子活 跃起来做无规则运动, 当氧化膜与 $\mathrm{NaCl}$ 溶液之间的电场强 度达到某一临界值时, 便出现点蚀现象并逐步增强。而用 包覆铸造工艺制备的试样却没有明显的腐蚀发生, 这是因 为用包覆制造工艺制备的涂层包覆在型芯表面, 熔融的 $\mathrm{Al}$ 合金金属液在浇入模具型腔与喷涂层接触的时候, $\mathrm{Al}$ 合金 金属液处于高温熔融状态, $\mathrm{Al}$ 合金金属液将喷涂层熔化从 而消除了部分气孔; 另外, 过热的 $\mathrm{Al}$ 合金金属液会渗入喷 涂层的空隙中, 随着金属液在空隙中的慢慢冷却与凝固, 使形成的小铸件填补了大部分的空隙, 其示意图如图5所 示。于是当包覆铸件进行 $5 \%$ 浓度的 $\mathrm{NaCl}$ 溶液浸泡试验时, 铸件表面不仅减少了大量的空隙与气孔, 而且还具有氧化
膜的保护, 使氯离子不能透过表面进入基体, 所以包覆铸 件的表面只有轻微的腐蚀。

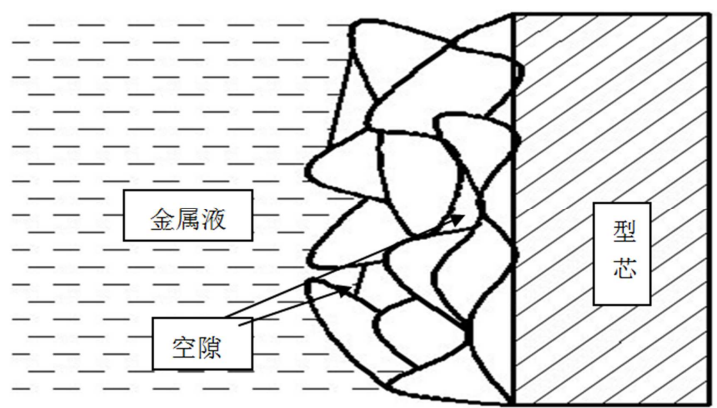

图5 包覆铸造过程示意图。

\section{5. 结论}

试验立足于型材与内界面包覆的研究, 探索了一种经 济、实用的内界面包覆型铸件的试验方法。利用该试验方 法制备了具有中间过渡层的Al-Al内界面包覆型铸件, 该 铸件的成功制备, 说明了该方法的可实施性。

对电弧喷涂 $\mathrm{Al}$ 层、基体 $\mathrm{Al}$ 及 $\mathrm{Al}-\mathrm{Al}$ 内界面包覆型铸 件三组试样进行了 $5 \%$ 浓度的 $\mathrm{NaCl}$ 浸泡实验。试验结果 表明, 电弧喷涂 $\mathrm{Al}$ 层、基体 $\mathrm{Al}$ 经 140 小时 $\mathrm{NaCl}$ 浸泡后, 气孔和间隙均有不同程度变大, 其中电弧喷涂 $\mathrm{A} 1$ 层变化 幅度最大, 而 Al-Al内界面包覆型铸件气孔大小和数量 基本没有变化, 说明耐蚀性较好。

\section{致谢}

项目：2017年重庆工商职业学院科学研究科研项目 (YB2017-13)

\section{参考文献}

[1] 刘慧芳, 高瑾, 李晓刚, 吴俊升. 高速电弧喷涂FeCrAl涂层 和 $3 \mathrm{Cr} 13$ 涂层的冲刷腐蚀性能 [J]. 中国腐蚀与防护学报, 2008,(04):225-230。

[2] 胡小刚. 319S铝合金高固相分数半固态压铸多尺度数值模 拟方法与实验研究 $[D]$. 北京: 北京科技大学, 2016。

[3] 华绍春, 王汉功, 汪刘应, 张武, 刘顾. 热喷涂技术的研究 进展 $[\mathrm{J}]$. 金属热处理, 2008,(05):82-87。

[4] 徐荣正, 宋刚, 刘黎明. 铝合金表面电弧喷涂铝涂层工艺与 性能[J]. 焊接学报, 2008,(06):109-112。

[5] 房元明, 徐骏, 高志华, 张志峰. 7075铝合金流变压铸数值 模拟及实验研究 [J]. 稀有金属, 2016,(06):283-286。

[6] A. Okuno, H. Kawano, J. Sun, M. Kurokawa, M. Nalkoka. Feasible Development of Soft-Switdled Sit Invener vith Load-adaptive Frequency-Tracking Control Scheme for Induction Heating [J]. IEEE-Transactions on industry Applications, 1998, 35(6): 713-718. 
[7] Mario Tama, Development of channel of core Type Iduction Furnaces [J], Industrial Heating, 1974(6): 43-45.

[8] D, V, AlexaIldro, solidification with a Quasiequilibrium Two-phase zone [J], Acta mater, 2001, 49(4): 759-764.

[9] Hashi M M, Otomo S, DeVdopmet of High Performance Roll by continuous Pouring Process for Cladding [J]. ISIJ, 1992, 11(32): 202-203.

[10] KISH ITAKE K. Thermal sp rayed Fe210Cr213P27C amorphous coatings possessing excelled corrosion resistance
[J]. Journal of Thermal Sp raing Thchnology, 1996, 5(4):476-481.

[11] 刘贵民, 徐滨士, 库日多夫斯基等. 电弧及高速电弧喷涂 $\mathrm{FeCrAl}$ 涂层的微观性能研究 [J]. 中国表面工程, 1999,44(3):11-14。

[12] 郭睿, 何汉军, 曾大新, 杜红. 液-液复合制备灰铸铁-球墨 铸铁双材料铸件的工艺研究 [J]. 铸造, 2017,(01):25-27。 